\title{
Undecidability of the Lambek Calculus with Subexponential and Bracket Modalities
}

\author{
Max Kanovich ${ }^{1}$, Stepan Kuznetsov ${ }^{2}$, and Andre Scedrov ${ }^{3}$ \\ 1 University College London and \\ National Research University Higher School of Economics (Moscow); \\ m.kanovich@qmul.ac.uk \\ 2 Steklov Mathematical Institute, RAS (Moscow); \\ sk@mi.ras.ru \\ 3 University of Pennsylvania (Philadelphia) and \\ National Research University Higher School of Economics (Moscow); \\ scedrov@math. upenn.edu
}

\begin{abstract}
The Lambek calculus is a well-known logical formalism for modelling natural language syntax. The original calculus covered a substantial number of intricate natural language phenomena, but only those restricted to the context-free setting. In order to address more subtle linguistic issues, the Lambek calculus has been extended in various ways. In particular, Morrill and Valentín (2015) introduce an extension with so-called exponential and bracket modalities. Their extension is based on a non-standard contraction rule for the exponential that interacts with the bracket structure in an intricate way. The standard contraction rule is not admissible in this calculus. In this paper we prove undecidability of the derivability problem in their calculus. We also investigate restricted decidable fragments considered by Morrill and Valentin and we show that these fragments belong to the NP class.
\end{abstract}

\section{Linguistic Introduction}

The Lambek calculus [23] is a substructural, non-commutative logical system (a variant of linear logic [15] in its intuitionistic non-commutative version [1]) that serves as the logical base for categorial grammars, a formalism that aims to describe natural language by means of logical derivability (see Buszkowski [9], Carpenter [11, Morrill [30], Moot and Retoré [28, etc). The idea of categorial grammar goes back to works of Ajdukiewicz [2] and Bar-Hillel [3], and afterwards it developed into several closely related frameworks, including combinatory categorial grammars (CCG, Steedman 39]), categorial dependency grammars (CDG, Dikovsky and Dekhtyar 12), and Lambek categorial grammars. A categorial grammar assigns syntactic categories (types) to words of the language. In the Lambek setting, types are constructed using two division operations, \and /, and the product, $\cdot$ Intuitively, $A \backslash B$ denotes the type of a syntactic object that lacks something of type $A$ on the left side to become an object of type $B ; B / A$ is symmetric; the product stands for concatenation. The Lambek calculus provides a system of rules for reasoning about syntactic types. 
For a quick example, consider the sentence "John loves Mary." Let "John" and "Mary" be of type $N$ (noun), and "loves" receive the type $(N \backslash S) / N$ of the transitive verb: it takes a noun from the left and a noun from the right, yielding a sentence, $S$. This sentence is judged as a grammatical one, because $N,(N \backslash S) / N, N \rightarrow S$ is a theorem in the Lambek calculus (and even in the Ajdukiewicz - Bar-Hillel logic for basic categorial grammars).

The Lambek calculus is capable of handling more complicated situations, including dependent clauses: "the girl whom John loves", parsed as $N$ using the following types: $N / C N, C N,(C N \backslash C N) /(S / N), N,(N \backslash S) / N \rightarrow N$ (here $C N$ stands for "common noun," a noun without an article), and coordination: "John loves Mary and Pete loves Kate," where "and" is $(S \backslash S) / S$.

There are, however, even more sophisticated cases for which the pure Lambek calculus is known to be insufficient (see, for example, 30, 28]). On the one hand, for a noun phrase like "the girl whom John met yesterday" it is problematic to find a correct type for "whom," since the dependent clause "John met yesterday" expects the lacking noun ("John met ... yesterday"; the "..." place is called gap) in the middle, and therefore is neither of type $S / N$ nor of type $N \backslash S$. This phenomenon is called medial extraction. On the other hand, the grammar sketched above generates, for example, * "the girl whom John loves Mary and Pete loves." The asterisk indicates ungrammaticality — but "John loves Mary and Pete loves" is yet of type $S / N$. To avoid this, one needs to block extraction from certain syntactic structures (e.g., compound sentences), called islands [38] 30].

These issues can be addressed by extending the Lambek calculus with extra connectives (that allow to derive more theorems) and also with a more sophisticated syntactic structure (that allows blocking unwanted derivations). In the next section, we follow Morrill and Valentín 30.33 and define an extension of the Lambek calculus with a subexponential modality (allows medial and also so-called parasitic extraction) and brackets (for creating islands).

\section{Logical Introduction}

In order to block ungrammatical extractions, such as discussed above, Morrill [29] and Moortgat 27] introduce an extension of the Lambek calculus with brackets that create islands. For the second issue, medial extraction, Morrill and Valentín [4]33] suggest using a modality which they call "exponential," in the spirit of Girard's exponential in linear logic 15. We rather use the term "subexponential," which is due to Nigam and Miller [34, since this modality allows only some of the structural rules (permutation and contraction, but not weakening). The difference from [34, however, is in the non-commutativity of the whole system and the non-standard nature of the contraction rule.

We consider $!_{\mathrm{b}} \mathbf{L}^{1}$, the Lambek calculus with the unit constant 24 , brackets, and a subexponential controlled by rules from 33 . The calculus $!_{\mathbf{b}} \mathbf{L}^{\mathbf{1}}$ is a conservative fragment of the $\mathbf{D b} !_{\mathbf{b}}$ system by Morrill and Valentín [33.

Due to brackets, the syntax of $!_{b} \mathbf{L}^{\mathbf{1}}$ is more involved than the syntax of a standard sequent calculus. Derivable objects are sequents of the form $\Pi \rightarrow A$. 
The antecedent $\Pi$ is a structure called meta-formula (or configuration); the succedent $A$ is a formula. Meta-formulae are built from formulae (types) using two metasyntactic operators: comma and brackets. The succedent $A$ is a formula. Formulae, in their turn, are built from primitive types (variables) $p_{1}, p_{2}, \ldots$ and the unit constant 1 using the Lambek's binary connectives: $\backslash, /$, and $\cdot$, and three unary connectives, \langle\rangle , [] $]^{-1}$, and !. The first two unary connectives operate brackets; the last one is the subexponential used for medial extraction.

Meta-formulae are denoted by capital Greek letters; $\Delta(\Gamma)$ stands for $\Delta$ with a designated occurrence of a meta-formula (in particular, formula) $\Gamma$. Metaformulae are allowed to be empty; the empty meta-formula is denoted by $\Lambda$.

The axioms of $\mathbf{l}_{\mathbf{b}} \mathbf{L}^{\mathbf{1}}$ are $A \rightarrow A$ and $\Lambda \rightarrow \mathbf{1}$, and the rules are as follows:

$$
\begin{array}{ccc}
\frac{\Gamma \rightarrow B}{\Delta(C / B, \Gamma) \rightarrow D}(/ \rightarrow) & \frac{\Gamma, B \rightarrow C}{\Gamma \rightarrow C / B}(\rightarrow /) & \frac{\Delta(A, B) \rightarrow D}{\Delta(A \cdot B) \rightarrow D}(\cdot \rightarrow) \\
\frac{\Gamma \rightarrow A \quad \Delta(C) \rightarrow D}{\Delta(\Gamma, A \backslash C) \rightarrow D}(\backslash \rightarrow) & \frac{A, \Gamma \rightarrow C}{\Gamma \rightarrow A \backslash C}(\rightarrow \backslash) & \frac{\Gamma_{1} \rightarrow A \quad \Gamma_{2} \rightarrow B}{\Gamma_{1}, \Gamma_{2} \rightarrow A \cdot B}(\rightarrow \cdot) \\
\frac{\Delta(\Lambda) \rightarrow A}{\Delta(\mathbf{1}) \rightarrow A}(\mathbf{1} \rightarrow) & \frac{\Delta([A]) \rightarrow C}{\Delta(\langle\rangle) \rightarrow C}(\langle\rangle) & \frac{\Pi \rightarrow A}{[\Pi] \rightarrow\langle\rangle A}(\rightarrow\langle\rangle) \\
\frac{\Gamma(A) \rightarrow B}{\Gamma(! A) \rightarrow B}(! \rightarrow) & \frac{\Delta(A) \rightarrow C}{\Delta\left(\left[[]^{-1} A\right]\right) \rightarrow C}\left([]^{-1} \rightarrow\right) & \frac{[\Pi] \rightarrow A}{\Pi \rightarrow[]^{-1} A}\left(\rightarrow[]^{-1}\right) \\
\frac{! A_{1}, \ldots, ! A_{n} \rightarrow A}{! A_{1}, \ldots, ! A_{n} \rightarrow ! A}(\rightarrow !) & \frac{\Delta\left(! A_{1}, \ldots, ! A_{n},\left[! A_{1}, \ldots, \ldots A_{n}, \Gamma\right]\right) \rightarrow B}{\Delta\left(! A_{1}, \ldots, ! A_{n}, \Gamma\right) \rightarrow B}\left(\operatorname{contr}_{\mathbf{b}}\right) \\
\frac{\Delta(! A, \Gamma) \rightarrow B}{\Delta(\Gamma, ! A) \rightarrow B}\left(\operatorname{perm}_{1}\right) & \frac{\Delta(\Gamma, ! A) \rightarrow B}{\Delta(! A, \Gamma) \rightarrow B}\left(\mathrm{perm}_{2}\right) & \frac{\Pi \rightarrow A \rightarrow \Delta(A) \rightarrow C}{\Delta(\Pi) \rightarrow C}(\mathrm{cut})
\end{array}
$$

The permutation rules $\left(\operatorname{perm}_{1,2}\right)$ for ! allow medial extraction. The relative pronoun "whom" now receives the type $(C N \backslash C N) /(S / ! N)$, and the noun phrase "the girl whom John met yesterday" now becomes derivable (the type for "yesterday" is $(N \backslash S) \backslash(N \backslash S)$, modifier of verb phrase):

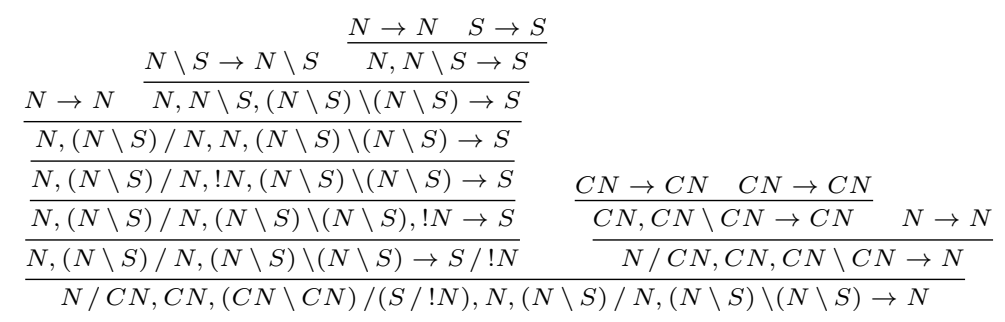

The permutation rule puts $! N$ to the correct place ("John met ... yesterday").

For brackets, consider the following ungrammatical example: * "the book which John laughed without reading." In the original Lambek calculus, it would be generated by the following derivable sequent:

$N / C N, C N,(C N \backslash C N) /(S / N), N, N \backslash S,((N \backslash S) \backslash(N \backslash S)) /(N \backslash S),(N \backslash S) / N \rightarrow N$. 
In the grammar with brackets, however, "without" receives the syntactic type []$^{-1}((N \backslash S) \backslash(N \backslash S)) /(N \backslash S)$, making the without-clause an island that cannot be penetrated by extraction. Thus, the following sequent is not derivable

$\left.N / C N, C N,(C N \backslash C N) /(S / N), N, N \backslash S,[]^{-1}((N \backslash S) \backslash(N \backslash S)) /(N \backslash S),(N \backslash S) / N\right] \rightarrow N$,

and the ungrammatical example gets ruled out.

Finally, the non-standard contraction rule, $\left(\operatorname{contr}_{\mathbf{b}}\right)$, that governs both ! and brackets, was designed for handling a more rare phenomenon called parasitic extraction. It appears in examples like "the paper that John signed without reading." Compare with the ungrammatical example considered before: now in the dependent clause there are two gaps, and one of them is inside an island ("John signed ... [without reading ...]"); both gaps are filled with the same $! N$ :

$$
\begin{aligned}
& N \backslash S \rightarrow N \backslash S \quad N, N \backslash S \rightarrow S \\
& \overline{N, N \backslash S,(N \backslash S) \backslash(N \backslash S) \rightarrow S} \\
& N \backslash S \rightarrow N \backslash S \quad \overline{N, N \backslash S,\left[[]^{-1}((N \backslash S) \backslash(N \backslash S))\right] \rightarrow S}
\end{aligned}
$$

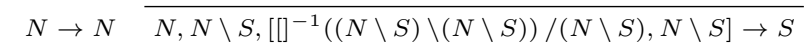

$$
\begin{aligned}
& \left.N \rightarrow N \quad N, N \backslash S,[]^{-1}((N \backslash S) \backslash(N \backslash S)) /(N \backslash S),(N \backslash S) / N, N\right] \rightarrow S \\
& N,(N \backslash S) / N, N,\left[[]^{-1}((N \backslash S) \backslash(N \backslash S)) /(N \backslash S),(N \backslash S) / N, N\right] \rightarrow S \\
& \overline{N,(N \backslash S) / N, ! N,\left[[]^{-1}((N \backslash S) \backslash(N \backslash S)) /(N \backslash S),(N \backslash S) / N, ! N\right] \rightarrow S} \\
& \overline{N,(N \backslash S) / N, ! N,\left[! N,[]^{-1}((N \backslash S) \backslash(N \backslash S)) /(N \backslash S),(N \backslash S) / N\right] \rightarrow S} \\
& N,(N \backslash S) / N, ! N,[]^{-1}((N \backslash S) \backslash(N \backslash S)) /(N \backslash S),(N \backslash S) / N \rightarrow S \\
& \overline{N,(N \backslash S) / N,[]^{-1}((N \backslash S) \backslash(N \backslash S)) /(N \backslash S),(N \backslash S) / N, ! N \rightarrow S} \\
& \overline{N,(N \backslash S) / N,[]^{-1}((N \backslash S) \backslash(N \backslash S)) /(N \backslash S),(N \backslash S) / N \rightarrow S / ! N} \quad N / C N, C N \rightarrow N \\
& N / C N, C N,(C N \backslash C N) /(S / ! N), N,(N \backslash S) / N,[]^{-1}((N \backslash S) \backslash(N \backslash S)) /(N \backslash S),(N \backslash S) / N \rightarrow N
\end{aligned}
$$

This construction allows potentially infinite recursion, nesting islands with parasitic extraction. On the other hand, ungrammatical examples, like * "the book that John gave to" with two gaps outside islands ("John gave ... to ...") are not derived with $\left(\operatorname{contr}_{\mathbf{b}}\right)$, but can be derived using the contraction rule in the standard, not bracket-aware form: $\frac{\Delta(! A, ! A) \rightarrow C}{\Delta(! A) \rightarrow C}$ (contr).

The system with (contr) instead of $\left(\operatorname{contr}_{\mathbf{b}}\right)$ is a conservative extension of its fragment without brackets. In an earlier paper 20 we show that the latter is undecidable. For $\left(\operatorname{contr}_{\mathbf{b}}\right)$, however, in the bracket-free fragment there are only permutation rules for !, and this fragment is decidable (in fact, it belongs to NP). Therefore, in contrast to [20, the undecidability proof in this paper (Section 5) crucially depends on brackets. On the other hand, in [20] we've also proved decidability of a fragment of a calculus with !, but without brackets. In the calculus considered in this paper, $!_{\mathrm{b}} \mathbf{L}^{\mathbf{1}}$, brackets control the number of $\left(\right.$ contr $\left._{\mathbf{b}}\right)$ applications, whence we are now able to show membership in NP for a different, broad fragment of $!_{\mathrm{b}} \mathbf{L}^{\mathbf{1}}$ (Section 6 ), which includes brackets.

It can be easily seen that the calculus with bracket modalities but without ! also belongs to the NP class. Moreover, as shown in [21, there exists even a polynomial algorithm for deriving formulae of bounded order (connective alternation and bracket nesting depth) in the calculus with brackets but without !. This algorithm uses proof nets, following the ideas of Pentus [36]. As opposed to [21, as we show here, in the presence of ! the derivability problem is undecidable. 
In short, 20 is about the calculus with !, but without brackets; 21] is about the calculus with brackets, but without !. This paper is about the calculus with both! and brackets, interacting with each other, governed by $\left(\operatorname{contr}_{\mathbf{b}}\right)$.

The rest of this paper is organised as follows. In Section 3 we formulate the cut elimination theorem for $!_{\mathrm{b}} \mathbf{L}^{1}$ and sketch the proof strategy; the detailed proof is placed in Appendix I. In Section 4 we define two intermediate calculi used in our undecidability proof. In Section 5 we prove the main result of this paper - the fact that $!_{\mathbf{b}} \mathbf{L}^{\mathbf{1}}$ is undecidable. This solves an open question posed by Morrill and Valentín [33] (the other open question from [33, undecidability for the case without brackets, is solved in our previous paper [20]). In Section 6 we consider a practically interesting fragment of $!_{\mathbf{b}} \mathbf{L}^{\mathbf{1}}$ for which Morrill and Valentín [33] present an exponential time algorithm and strengthen their result by proving an NP upper bound for the derivability problems in this fragments. Section 7 is for conclusion and future research.

\section{Cut Elimination in $!_{\mathrm{b}} \mathbf{L}^{1}$}

Cut elimination is a natural property that one expects a decent logical system to have. For example, cut elimination entails the subformula property: each formula that appears somewhere in the cut-free derivation is a subformula of the goal sequent. (Note that for meta-formulae this doesn't hold, since brackets get removed by applications of some rules, namely, $(\langle\rangle \rightarrow),\left(\rightarrow[]^{-1}\right)$, and $\left(\operatorname{contr}_{\mathbf{b}}\right)$.)

Theorem 1 is claimed in [33, but without a detailed proof. In this section we give a sketch of the proof strategy; the complete proof is in Appendix I.

For the original Lambek calculus cut elimination was shown by Lambek 23] and goes straightforwardly by induction; Moortgat [27] extended Lambek's proof to the Lambek calculus with brackets (but without !). It is well-known, however, that in the presence of a contraction rule direct induction doesn't work. Therefore, one needs to use more sophisticated cut elimination strategies.

The standard strategy, going back to Gentzen's Hauptsatz [14, replaces the cut (Schnitt) rule with a more general rule called mix (Mischung). Mix is a combination of cut and contraction, and this more general rule can be eliminated by straightforward induction. For linear logic with the exponential obeying standard rules, cut elimination is due to Girard [15; ; a detailed exposition of the cut elimination procedure using mix is presented in [25. Appendix A].

For $!_{\mathbf{b}} \mathbf{L}^{\mathbf{1}}$, however, due to the subtle nature of the contraction rule, (contr $\left.\mathbf{b}\right)$, formulating the mix rule is problematic. Therefore, here we follow another strategy, "deep cut elimination" by Braüner and de Paiva [5] 6]; similar ideas are also used in [7] and [13. As usually, we eliminate one cut, and then proceed by induction.

Lemma 1. Let $\Delta(\Pi) \rightarrow C$ be derived from $\Pi \rightarrow A$ and $\Delta(A) \rightarrow C$ using the cut rule, and $\Pi \rightarrow A$ and $\Delta(A) \rightarrow C$ have cut-free derivations $\mathscr{D}_{\text {left }}$ and $\mathscr{D}_{\text {right }}$. Then $\Delta(\Pi) \rightarrow C$ also has a cut-free derivation. 
We proceed by nested induction on two parameters: (1) the complexity $\kappa$ of the formula $A$ being cut; (2) the total number $\sigma$ of rule applications in $\mathscr{D}_{\text {left }}$ and $\mathscr{D}_{\text {right }}$. Induction goes smoothly for all cases, except the case where the last rule in $\mathscr{D}_{\text {left }}$ is $(\rightarrow !)$ and the last rule in $\mathscr{D}_{\text {right }}$ is $\left(\operatorname{contr}_{\mathbf{b}}\right)$ :

$$
\frac{\frac{! \Pi \rightarrow A}{! \Pi \rightarrow ! A}(\rightarrow !) \frac{\Delta\left(! \Phi_{1}, ! A, ! \Phi_{2},\left[! \Phi_{1}, ! A, ! \Phi_{2}, \Gamma\right]\right) \rightarrow C}{\Delta\left(! \Phi_{1}, ! A, ! \Phi_{2}, \Gamma\right) \rightarrow C}(\text { cut })}{\Delta\left(! \Phi_{1}, ! \Pi, ! \Phi_{2}, \Gamma\right) \rightarrow C}\left(\text { contr }_{\mathbf{b}}\right)
$$

(Here $! \Phi$ stands for $! F_{1}, \ldots, ! F_{m}$, if $\Phi=F_{1}, \ldots, F_{m}$.) The naïve attempt,

$$
\frac{! \Pi \rightarrow ! A \quad \frac{! \Pi \rightarrow ! A \quad \Delta\left(! \Phi_{1}, ! A, ! \Phi_{2},\left[! \Phi_{1}, ! A, ! \Phi_{2}, \Gamma\right]\right) \rightarrow C}{\Delta\left(! \Phi_{1}, ! A, ! \Phi_{2},\left[! \Phi_{1}, ! A, ! \Phi_{2}, \Gamma\right]\right) \rightarrow C}}{\frac{\Delta\left(! \Phi_{1}, ! \Pi, ! \Phi_{2},\left[! \Phi_{1}, ! \Pi, ! \Phi_{2}, \Gamma\right]\right) \rightarrow C}{\Delta\left(! \Phi_{1}, ! \Pi, ! \Phi_{2}, \Gamma\right) \rightarrow C}\left(\operatorname{contr}_{\mathbf{b}}\right)} \text { (cut) }
$$

fails, since for the lower (cut) the $\kappa$ parameter is the same, and $\sigma$ is uncontrolled. Instead of that, the "deep" cut elimination strategy goes inside $\mathscr{D}_{\text {right }}$ and traces the active $! A$ occurrences up to the applications of $(! \rightarrow)$ which introduced them. Instead of these applications we put (cut) with the left premise, $! \Pi \rightarrow A$, and replace ! $A$ with $! \Pi$ down the traces. The new (cut) instances have a smaller $\kappa$ parameter $(A$ is simpler than $! A)$ and can be eliminated by induction.

Theorem 1. Every sequent derivable in $!_{\mathbf{b}} \mathbf{L}^{\mathbf{1}}$ has a derivation without (cut).

\section{Calculi Without Brackets: ! $\mathrm{L}^{1}, !_{\mathrm{w}} \mathrm{L}^{1}, \mathrm{~L}^{1}$}

In this section we consider more traditional versions of the Lambek calculus with ! that don't include bracket modalities. This is needed as a technical step in our undecidability proof (Section 5). Types (formulae) of these calculi are built from primitive types using Lambek's connectives, $\backslash, /$, and ·, and the subexponential, !. Unlike in $!_{\mathrm{b}} \mathbf{L}^{\mathbf{1}}$, meta-formulae now are merely linearly ordered sequences of formulae (possibly empty), and we can write $\Delta_{1}, \Pi, \Delta_{2}$ instead of $\Delta(\Pi)$.

First we define the calculus $! \mathbf{L}^{1}$. It includes the standard axioms and rules for Lambek connectives and the unit constant - see the rules of $!_{\mathrm{b}} \mathbf{L}^{\mathbf{1}}$ in Section 2 . For the subexponential modality, !, introduction rules, $(! \rightarrow)$ and $(\rightarrow !)$, and permutation rules are also the same as in $!_{\mathbf{b}} \mathbf{L}^{\mathbf{1}}$, with the natural modification due to a simpler antecedent syntax. The contraction rule, however, is significantly different, since now it is not controlled by brackets:

$$
\frac{\Delta_{1}, ! A, ! A, \Delta_{2} \rightarrow B}{\Delta_{1}, ! A, \Delta_{2} \rightarrow B}(\mathrm{contr})
$$

The full set of axioms and rules of $\mathbf{~} \mathbf{L}^{\mathbf{1}}$ is presented in Appendix II.

This calculus $\mathbf{~} \mathbf{L}^{\mathbf{1}}$ is a conservative fragment of $\mathbf{D b}$ !, also by Morrill and Valentín [33. This system could also be used for modelling medial and parasitic 
extraction, but is not as fine-grained as the bracketed system, being able to derive ungrammatical examples like * "the paper that John sent to" (see Section 2).

In order to construct a mapping of $!_{\mathbf{b}} \mathbf{L}^{\mathbf{1}}$ into $! \mathbf{L}^{\mathbf{1}}$, we define the bracketforgetting projection (BFP) of formulae and meta-formulae that removes all brackets and bracket modalities $\left([]^{-1}\right.$ and \langle\rangle$)$. The BFP of a formula is again a formula, but in the language without []$^{-1}$ and \langle\rangle ; the BFP of a meta-formula is a sequence of formulae. The following lemma is proved by induction on derivation.

Lemma 2. If $!_{\mathrm{b}} \mathbf{L}^{\mathbf{1}} \vdash \Delta \rightarrow C$, then $\mathbf{l}^{\mathbf{1}} \vdash \operatorname{BFP}(\Delta) \rightarrow \operatorname{BFP}(C)$.

Note that the opposite implication doesn't hold, i.e., this mapping is not conservative. Also, $! \mathbf{L}^{\mathbf{1}}$ is not a conservative fragment of $!_{\mathrm{b}} \mathbf{L}^{\mathbf{1}}$ : in the fragment of $!_{\mathrm{b}} \mathbf{L}^{1}$ without brackets contraction is not admissible.

The second calculus is $!_{w} \mathbf{L}^{1}$, obtained from $! \mathbf{L}^{1}$ by adding weakening for !:

$$
\frac{\Delta_{1}, \Delta_{2} \rightarrow C}{\Delta_{1}, ! A, \Delta_{2} \rightarrow C} \text { (weak) }
$$

In $!_{w} \mathbf{L}^{1}$, the ! connective is equipped with a full set of structural rules (permutation, contraction, and weakening), i.e., it is the exponential of linear logic [15].

The cut rule in $\mathbf{L}^{\mathbf{1}}$ and $!_{\mathrm{w}} \mathbf{L}^{\mathbf{1}}$ can be eliminated by the same "deep" strategy as for $!_{b} \mathbf{L}^{\mathbf{1}}$. On the other hand, since the contraction rule in these calculi is standard, one can also use the traditional way with mix, like in [25, Appendix A].

Finally, if we remove! with all its rules, we get the Lambek calculus with the unit constant [24]. We denote it by $\mathbf{L}^{\mathbf{1}}$.

\section{$5 \quad$ Undecidability of $!_{\mathrm{b}} \mathrm{L}^{1}$}

The main result of this paper is:

Theorem 2. The derivability problem for $!_{\mathbf{b}} \mathbf{L}^{\mathbf{1}}$ is undecidable.

As a by-product of our proof we also obtain undecidability of $\mathbf{~} \mathbf{L}^{\mathbf{1}}$, which was proved in 20 by a different method. We also obtain undecidability of $!_{w} \mathbf{L}^{\mathbf{1}}$, which also follows from the results of [25, as shown in [17] and [16.

We prove Theorem 2 by encoding derivations in generative grammars, or semi-Thue [40] systems. A generative grammar is a quadruple $G=\langle N, \Sigma, P, s\rangle$, where $N$ and $\Sigma$ are two disjoint alphabets, $s \in N$ is the starting symbol, and $P$ is a finite set of productions (rules) of the form $\alpha \Rightarrow \beta$, where $\alpha$ and $\beta$ are words over $N \cup \Sigma$. The production can be applied in the following way: $\eta \alpha \theta \Rightarrow_{G} \eta \beta \theta$, where $\eta$ and $\theta$ are arbitrary (possibly empty) words over $N \cup \Sigma$. The language generated by $G$ is the set of all words $\omega$ over $\Sigma$, such that $s \Rightarrow_{G}^{*} \omega$, where $\Rightarrow_{G}^{*}$ is the reflexive-transitive closure of $\Rightarrow_{G}$.

We use the following classical result by Markov [26] and Post [37.

Theorem 3. There exists a generative grammar $G$ that generates an algorithmically undecidable language. 26] 37. 
In our presentation for every production $(\alpha \Rightarrow \beta) \in P$ we require $\alpha$ and $\beta$ to be non-empty. This class still includes an undecidable language (cf. 10]).

Further we use two trivial lemmas about derivations in a generative grammar:

Lemma 3. If $\alpha_{1} \Rightarrow_{G}^{*} \beta_{1}$ and $\alpha_{2} \Rightarrow_{G}^{*} \beta_{2}$, then $\alpha_{1} \alpha_{2} \Rightarrow_{G}^{*} \beta_{1} \beta_{2}$.

Lemma 4. If $\alpha \Rightarrow_{G}^{*} \beta$ and $\gamma \Rightarrow_{G}^{*} \eta \alpha \theta$, then $\gamma \Rightarrow_{G}^{*} \eta \beta \theta$.

The second ingredient we need for our undecidability proof is the concept of theories over $\mathbf{L}^{\mathbf{1}}$. Let $\mathcal{T}$ be a finite set of sequents in the language of $\mathbf{L}^{\mathbf{1}}$. Then $\mathbf{L}^{\mathbf{1}}+\mathcal{T}$ is the calculus from $\mathbf{L}^{\mathbf{1}}$ by adding sequents from $\mathcal{T}$ as extra axioms.

In general, the cut rule in $\mathbf{L}^{\mathbf{1}}+\mathcal{T}$ is not eliminable. However, the standard cut elimination procedure (see [23]) yields the following cut normalization lemma:

Lemma 5. If a sequent is derivable in $\mathbf{L}^{\mathbf{1}}+\mathcal{T}$, then this sequent has a derivation in which every application of (cut) has a sequent from $\mathcal{T}$ as one of its premises.

This lemma yields a weak version of the subformula property:

Lemma 6. If $\mathbf{L}^{1}+\mathcal{T} \vdash \Pi \rightarrow A$, and both $\Pi \rightarrow A$ and $\mathcal{T}$ include no occurrences of $\backslash, /$, and $\mathbf{1}$, then there is a derivation of $\Pi \rightarrow A$ in $\mathbf{L}^{\mathbf{1}}+\mathcal{T}$ that includes no occurrences of $\backslash, /$, and $\mathbf{1}$.

The third core element of the construction is the (inst) rule which allows to place a specific formula $A$ into an arbitrary place in the sequent.

Lemma 7. The following rule is admissible in $!_{\mathbf{b}} \mathbf{L}^{\mathbf{1}}$ :

$$
\frac{\Delta_{1}, ![]^{-1} A, \Delta_{2}, A, \Delta_{3} \rightarrow C}{\Delta_{1}, ![]^{-1} A, \Delta_{2}, \Delta_{3} \rightarrow C} \text { (inst) }
$$

Proof.

$$
\frac{\frac{\Delta_{1}, ![]^{-1} A, \Delta_{2}, A, \Delta_{3} \rightarrow C}{\left.\Delta_{1}, ![]^{-1} A, \Delta_{2},[]^{-1} A\right], \Delta_{3} \rightarrow C}}{\frac{\Delta_{1}, ![]^{-1} A, \Delta_{2},\left[![]^{-1} A\right], \Delta_{3} \rightarrow C}{\Delta_{1}, \Delta_{2}, ![]^{-1} A,\left[![]^{-1} A\right], \Delta_{3} \rightarrow C}}\left(\begin{array} { l } 
{ ( ! \rightarrow ) } \\
{ \frac { \Delta _ { 1 } , \Delta _ { 2 } , ! [ ] ^ { - 1 } A , \Delta _ { 3 } \rightarrow C } { \Delta _ { 1 } , ! [ ] ^ { - 1 } A , \Delta _ { 2 } , \Delta _ { 3 } \rightarrow C } ( \operatorname { p e r m } _ { 1 } ) }
\end{array} \left(\begin{array}{l}
\left.\operatorname{contr}_{\mathbf{b}}\right) \\
\left.\operatorname{corm}_{2}\right)
\end{array}\right.\right.
$$

Now we are ready to prove Theorem 2. Let $G=\langle N, \Sigma, P, s\rangle$ be the grammar provided by Theorem 3 , and the set of variables include $N \cup \Sigma$. We convert productions of $G$ into Lambek formulae in the following natural way:

$\mathcal{B}_{G}=\left\{\left(u_{1} \cdot \ldots \cdot u_{k}\right) /\left(v_{1} \cdot \ldots \cdot v_{m}\right) \mid\left(u_{1} \ldots u_{k} \Rightarrow v_{1} \ldots v_{m}\right) \in P\right\}$.

For $\mathcal{B}_{G}=\left\{B_{1}, \ldots, B_{n}\right\}$, we define the following sequences of formulae:

$$
\begin{array}{lll}
\Gamma_{G}=! B_{1}, \ldots, ! B_{n}, & \Phi_{G}=!\left(\mathbf{1} /\left(! B_{1}\right)\right), \ldots, !\left(\mathbf{1} /\left(! B_{n}\right)\right), \\
\widetilde{\Gamma}_{G}=![]^{-1} B_{1}, \ldots, ![]^{-1} B_{n}, & \widetilde{\Phi}_{G}=!\left(\mathbf{1} /\left(![]^{-1} B_{1}\right)\right), \ldots, !\left(\mathbf{1} /\left(![]^{-1} B_{n}\right)\right) .
\end{array}
$$

(Since in all calculi we have permutation rules for formulae under !, the ordering of $\mathcal{B}_{G}$ doesn't matter.) We also define a theory $\mathcal{T}_{G}$ associated with $G$, as follows: $\mathcal{T}_{G}=\left\{v_{1}, \ldots, v_{m} \rightarrow u_{1} \cdot \ldots \cdot u_{k} \mid\left(u_{1} \ldots u_{k} \Rightarrow v_{1} \ldots v_{m}\right) \in P\right\}$. 
Lemma 8. The following are equivalent:

1. $s \Rightarrow_{G}^{*} a_{1} \ldots a_{n}$ (i.e., $a_{1} \ldots a_{n}$ belongs to the language defined by $G$ );

2. $!_{\mathrm{b}} \mathbf{L}^{\mathbf{1}} \vdash \widetilde{\Phi}_{G}, \widetilde{\Gamma}_{G}, a_{1}, \ldots, a_{n} \rightarrow s$;

3. $! \mathbf{L}^{\mathbf{1}} \vdash \Phi_{G}, \Gamma_{G}, a_{1}, \ldots, a_{n} \rightarrow s$;

4. $!_{\mathrm{w}} \mathbf{L}^{\mathbf{1}} \vdash \Gamma_{G}, a_{1}, \ldots, a_{n} \rightarrow s$;

5. $\mathbf{L}^{\mathbf{1}}+\mathcal{T}_{G} \vdash a_{1}, \ldots, a_{n} \rightarrow s$.

Proof. $1 \Rightarrow 2$ Proceed by induction on $\Rightarrow_{G}^{*}$. The base case is handled as follows:

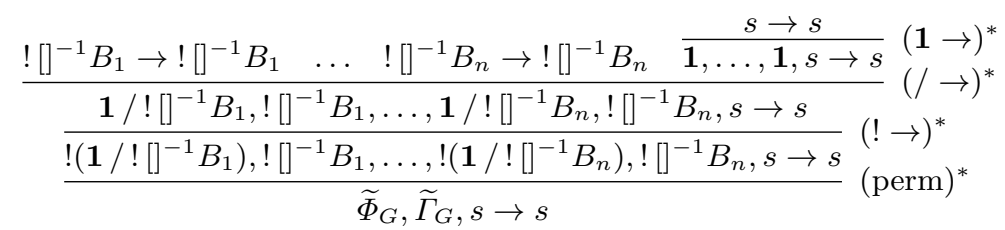

For the induction step let the last production be $u_{1} \ldots u_{k} \Rightarrow v_{1} \ldots v_{m}$, i.e., $s \Rightarrow_{G}^{*} \eta u_{1} \ldots u_{k} \theta \Rightarrow_{G} \eta v_{1} \ldots v_{m} \theta$.

Then, since ! [ ] $]^{-1}\left(\left(u_{1} \cdot \ldots \cdot u_{k}\right) /\left(v_{1} \cdot \ldots \cdot v_{m}\right)\right)$ is in $\widetilde{\Gamma}_{G}$, we enjoy the following:

$$
\frac{\frac{v_{1} \rightarrow v_{1} \ldots v_{m} \rightarrow v_{m}}{v_{1}, \ldots, v_{m} \rightarrow v_{1} \cdot \ldots \cdot v_{m}}(\rightarrow \cdot)^{*} \frac{\widetilde{\Phi}_{G}, \widetilde{\Gamma}_{G}, \eta, u_{1}, \ldots, u_{k}, \theta \rightarrow s}{\widetilde{\Phi}_{G}, \widetilde{\Gamma}_{G}, \eta, u_{1} \cdot \ldots \cdot u_{k}, \theta \rightarrow s}(\cdot \rightarrow)^{*}}{\frac{\widetilde{\Phi}_{G}, \widetilde{\Gamma}_{G}, \eta,\left(u_{1} \cdot \ldots \cdot u_{k}\right) /\left(v_{1} \cdot \ldots \cdot v_{m}\right), v_{1}, \ldots, v_{m}, \theta \rightarrow s}{\widetilde{\Phi}_{G}, \widetilde{\Gamma}_{G}, \eta, v_{1}, \ldots, v_{m}, \theta \rightarrow s} \text { (inst) }}(/)
$$

Here $\widetilde{\Phi}_{G}, \widetilde{\Gamma}_{G}, \eta, u_{1}, \ldots, u_{k}, \theta \rightarrow s$ is derivable in $!_{\mathbf{b}} \mathbf{L}^{\mathbf{1}}$ by induction hypothesis, and the (inst) rule is admissible due to Lemma 7.

$2 \Rightarrow 3$ Immediately by Lemma 2 since $\Phi_{G}=\operatorname{BFP}\left(\widetilde{\Phi}_{G}\right)$ and $\Gamma_{G}=\operatorname{BFP}\left(\widetilde{\Gamma}_{G}\right)$.

3a4 For each formula ! $\left(\mathbf{1} / ! B_{i}\right)$ from $\Phi_{G}$ the sequent $\Lambda \rightarrow !\left(\mathbf{1} / ! B_{i}\right)$ is derivable in $!_{\mathrm{w}} \mathbf{L}^{\mathbf{1}}$ by consequent application of (weak), $(\rightarrow /)$, and $(\rightarrow !)$ to the $\Lambda \rightarrow \mathbf{1}$ axiom. The sequent $\Phi_{G}, \Gamma_{G}, a_{1}, \ldots, a_{n} \rightarrow s$ is derivable in $\mathbf{! L}^{\mathbf{1}}$ and therefore in $!_{\mathrm{w}} \mathbf{L}^{1}$, and applying (cut) for each formula of $\Phi_{G}$ yields $\Gamma_{G}, a_{1}, \ldots, a_{n} \rightarrow s$.

$4 \Rightarrow 5$ In this part of our proof we follow [25] and [17. Consider the derivation of $\Gamma_{G}, a_{1}, \ldots, a_{n} \rightarrow s$ in $!_{\mathbf{w}} \mathbf{L}^{1}$ (recall that by default all derivations are cut-free) and remove all the formulae of the form $! B$ from all sequents in this derivation. After this transformation the rules not operating with! remain valid. Applications of $\left(\right.$ perm $\left._{i}\right)$, (weak), and (contr) do not alter the sequent. The $(\rightarrow !)$ rule is never applied in the original derivation, since our sequents never have formulae of the form $! B$ in their succedents. Finally, an application of $(! \rightarrow)$,

$$
\frac{\Delta_{1},\left(u_{1} \cdot \ldots \cdot u_{k}\right) /\left(v_{1} \cdot \ldots \cdot v_{m}\right), \Delta_{2} \rightarrow C}{\Delta_{1}, \Delta_{2} \rightarrow C},
$$

is simulated in $\mathbf{L}^{\mathbf{1}}+\mathcal{T}_{G}$ in the following way:

$$
\begin{aligned}
& \frac{v_{1}, \ldots, v_{m} \rightarrow u_{1} \cdot \ldots \cdot u_{k}}{v_{1} \cdot \ldots \cdot v_{m} \rightarrow u_{1} \cdot \ldots \cdot u_{k}}(\cdot \rightarrow)^{*} \\
& \frac{\frac{v_{1} \cdot \ldots \cdot v_{m} \rightarrow u_{1} \cdot \ldots \cdot u_{k}}{\Lambda \rightarrow\left(u_{1} \cdot \ldots \cdot u_{k}\right) /\left(v_{1} \cdot \ldots \cdot v_{m}\right)}(\rightarrow /) \quad \Delta_{1},\left(u_{1} \cdot \ldots \cdot u_{k}\right) /\left(v_{1} \cdot \ldots \cdot v_{m}\right), \Delta_{2} \rightarrow C}{\Delta_{1}, \Delta_{2} \rightarrow C} \text { (cut) }
\end{aligned}
$$


$5 \Rightarrow 1$ In this part we follow 25]. Let $\mathbf{L}^{1}+\mathcal{T}_{G} \vdash a_{1}, \ldots, a_{n} \rightarrow s$. By Lemma 6 this sequent has a derivation without occurrences of $\backslash, /$, and $\mathbf{1}$. In other words, all formulae in this derivation are built from variables using only the product. Since it is associative, we can omit parenthesis in the formulae; we shall also omit the "."s. The rules used in this derivation can now be written as follows:

$$
\frac{\beta_{1} \rightarrow \alpha_{1} \quad \beta_{2} \rightarrow \alpha_{2}}{\beta_{1} \beta_{2} \rightarrow \alpha_{1} \alpha_{2}}(\rightarrow \cdot) \quad \frac{\beta \rightarrow \alpha \quad \eta \alpha \theta \rightarrow \gamma}{\eta \beta \theta \rightarrow \gamma} \text { (cut) }
$$

The $(\cdot \rightarrow)$ rule is trivial. The axioms are productions of $G$ with the arrows inversed, and $\alpha \rightarrow \alpha$. By induction, using Lemmas 3 and 4 , we show that if $\beta \rightarrow \alpha$ is derivable using these rules and axioms, then $\alpha \Rightarrow_{G}^{*} \beta$. Now the derivability of $a_{1}, \ldots, a_{n} \rightarrow s$ implies $s \Rightarrow_{G}^{*} a_{1} \ldots a_{n}$.

Lemma 8 and Theorem 3 conclude the proof of Theorem 2

\section{A Decidable Fragment}

The undecidability results from the previous section are somewhat unfortunate, since the new operations added to $\mathbf{L}^{\mathbf{1}}$ have good linguistic motivations [33] 30. As a compensation, in this section we show NP-decidability for a substantial fragment of $!_{\mathrm{b}} \mathbf{L}^{\mathbf{1}}$, introduced by Morrill and Valentín [33] (see Definition 1 below). This complexity upper bound is tight, since the original Lambek calculus is already known to be NP-complete [35]. Notice that Morrill and Valentín present an exponential time algorithm for deciding derivability in this fragment; this algorithm was implemented as part of a parser called CatLog [31].

First we recall the standard notion of polarity of occurrences of subformulae in a formula. Every formula occurs positively in itself; subformula polarities get inverted (positive becomes negative and vice versa) when descending into denominators of $\backslash$ and / and also for the left-hand side of the sequent; brackets and all unary operations don't change polarity. All inference rules of $!_{\mathbf{b}} \mathbf{L}^{\mathbf{1}}$ respect polarity: a positive (resp., negative) occurrence of a subformula in the premise(s) of the rule translates into a positive (resp., negative) occurrence in the goal.

Definition 1. An $!_{\mathrm{b}} \mathbf{L}^{1}$-sequent $\Gamma \rightarrow B$ obeys the bracket non-negative condition, if any negative occurrence of a subformula of the form $! A$ in $\Gamma \rightarrow B$ includes neither a positive occurrence of a subformula of the form []$^{-1} C$, nor a negative occurrence of a subformula of the form \langle\rangle $C$.

Note that sequents used in our undecidability proof are exactly the minimal violations of this bracket non-negative condition.

Theorem 4. The derivability problem in $!_{\mathbf{b}} \mathbf{L}^{\mathbf{1}}$ for sequents that obey the bracket non-negative condition belongs to the NP class.

Derivations in $!_{\mathbf{b}} \mathbf{L}^{\mathbf{1}}$ are a bit incovenient for complexity estimations, since redundant applications of permutation rules could make the proof arbitrarily 
large without increasing its "real" complexity. In order to get rid of that, we introduce a generalised form of permutation rule:

$$
\frac{\Delta_{0}, ! A_{1}, \Delta_{1}, ! A_{2}, \Delta_{2}, \ldots, \Delta_{k-1}, ! A_{k}, \Delta_{k} \rightarrow C}{\Delta_{0}^{\prime}, ! A_{i_{1}}, \Delta_{1}^{\prime}, ! A_{i_{2}}, \Delta_{2}^{\prime}, \ldots, \Delta_{i_{k-1}}^{\prime}, ! A_{i_{k}}, \Delta_{i_{k}}^{\prime} \rightarrow C}(\text { perm })^{*}
$$

where the sequence $\Delta_{0}^{\prime}, \ldots, \Delta_{k}^{\prime}$ coincides with $\Delta_{0}, \ldots, \Delta_{k}$, and $\left\{i_{1}, \ldots, i_{k}\right\}=$ $\{1, \ldots, k\}$. Obviously, (perm)* is admissible in $!_{\mathrm{b}} \mathbf{L}^{\mathbf{1}}$, and it subsumes $\left(\operatorname{perm}_{1,2}\right)$, so further we consider a formulation of $!_{\mathbf{b}} \mathbf{L}^{\mathbf{1}}$ with $(\text { perm })^{*}$ instead of $($ perm 1,2$)$. Several consecutive applications of (perm)* can be merged into one. We call a derivation normal, if it doesn't contain consecutive applications of (perm)*. If a sequent is derivable in $!_{\mathbf{b}} \mathbf{L}^{\mathbf{1}}$, then it has a normal cut-free derivation.

Lemma 9. Every normal cut-free derivation of a sequent that obeys bracket non-negative restriction is of quadratic size (number of rule applications) w.r.t. the size of the goal sequent.

Proof. Let us call ( contr $\left._{\mathbf{b}}\right)$ and (perm)* structural rules, and all others logical.

First, we track all pairs of brackets that occur in this derivation. Pairs of brackets are in one-to-one correspondence with applications of $\left([]^{-1} \rightarrow\right)$ or $(\rightarrow\langle\rangle)$ rules that introduce them. Then a pair of brackets either traces down to the goal sequent, or gets destroyed by an application of $(\langle\rangle \rightarrow),\left(\rightarrow[]^{-1}\right)$, or $\left(\operatorname{contr}_{\mathbf{b}}\right)$. Therefore, the total number of $\left(\operatorname{contr}_{\mathbf{b}}\right)$ applications is less or equal to the number of $\left([]^{-1} \rightarrow\right)$ and $(\rightarrow\langle\rangle)$ applications. Each $\left([]^{-1} \rightarrow\right)$ application introduces a negative occurrence of a []$^{-1} C$ formula; each $(\rightarrow\langle\rangle)$ occurrence introduces a positive occurrence of a \langle\rangle $C$ formula. Due to the bracket non-negative condition these formulae are never contracted (i.e., could not occur in a! $A$ to which $\left(\operatorname{contr}_{\mathbf{b}}\right)$ is applied), and therefore they trace down to distinct subformula occurrences in the goal sequent. Hence, the total number of $\left(\operatorname{contr}_{\mathbf{b}}\right)$ applications is bounded by the number of subformulae of a special kind in the goal sequent, in other words, it is bounded by the size of the sequent.

Second, we bound the number of logical rules applications. Each logical rule introduces exactly one connective occurrence. Such an occurrence traces down either to a connective occurrence in the goal sequent, or to an application of $\left(\operatorname{contr}_{\mathbf{b}}\right)$ that merges this occurrence with the corresponding occurrence in the other! $A$. If $n$ is the size of the goal sequent, then the first kind of occurrences is bounded by $n$; for the second kind, notice that each application of ( $\left.\operatorname{contr}_{\mathbf{b}}\right)$ merges not more than $n$ occurrences (since the size of the formula being contracted, ! $A$, is bounded by $n$ due to the subformula property), and the total number of $\left(\operatorname{contr}_{\mathbf{b}}\right)$ applications is also bounded by $n$. Thus, we get a quadratic bound for the number of logical rule applications.

Third, the derivation is a tree with binary branching, so the number of leafs (axioms instances) in this tree is equal to the number of branching points plus one. Each branching point is an application of a logical rule (namely, $(\backslash \rightarrow$ ), $(/ \rightarrow)$, or $(\rightarrow \cdot))$. Hence, the number of axiom instances is bounded quadratically.

Finally, the number of (perm)* applications is also quadratically bounded, since each application of (perm)* in a normal proof is preceded by an application of another rule or by an axiom instance. 
Proof (of Theorem 4). The normal derivation of a sequent obeying the bracket non-negative condition is an NP-witness for derivability: it is of polynomial size, and correctness is checked in linear time (w.r.t. the size of the derivation).

For the case without brackets, $! \mathbf{L}^{\mathbf{1}}$, considered in our earlier paper [20, the NP-decidable fragment is substantially smaller. Namely, it includes only sequents in which ! can be applied only to variables. Indeed, as soon as we allow formulae of implication nesting depth at least 2 under !, the derivablity problem for $\mathbf{~} \mathbf{L}^{\mathbf{1}}$ becomes undecidable 20 . In contrast to $! \mathbf{L}^{\mathbf{1}}$, in $!_{\mathbf{b}} \mathbf{L}^{\mathbf{1}}$, due to the non-standard contraction rule, brackets control the number of $\left(\operatorname{contr}_{\mathbf{b}}\right)$ applications in the proof, and this allows to construct an effective decision algorithm for derivability of a broad class of sequents, where, for example, any formulae without bracket modalities can be used under !. Essentially, the only problematic situation, that gives rise to undecidability (Theorem 2), is the construction where one forcedly removes the brackets that appear in the $\left(\operatorname{contr}_{\mathbf{b}}\right)$ rule, i.e., uses constructions like! []$^{-1} B$ (as in our undecidability proof). The idea of the bracket non-negative condition is to rule out such situations while keeping all other constructions allowed, as they don't violate decidability [33.

\section{Conclusions and Future Work}

In this paper we study an extension of the Lambek calculus with subexponential and bracket modalities. Bracket modalities were introduced by Morrill 29] and Moortgat [27] in order to represent the linguistic phenomenon of islands [38]. The interaction of subexponential and bracket modalities was recently studied by Morrill and Valentín [33] in order to represent correctly the phenomenon of medial and parasitic extraction 38, 4. We prove that the calculus of Morrill and Valentín is undecidable, thus solving a problem left open in [33. Morrill and Valentín also considered the so-called bracket non-negative fragment of this calculus, for which they presented an exponential time derivability decision procedure. We improve their result by showing that this problem is in NP.

Our undecidability proof is based on encoding semi-Thue systems by means of sequents that lie just outside the bracket non-negative fragment. More precisely, the formulae used in our encoding are of the from ! [ ] ${ }^{-1} A$, where $A$ is a pure Lambek formula of order 2. It remains for further investigation whether these formulae could be simplified.

Our undecidability proof could be potentially made stronger by restricting the language. Now we use three connectives of the original Lambek calculus: $/, \cdot$, and $\mathbf{1}$, plus [ ] ${ }^{-1}$ and !. One could get rid of $\mathbf{1}$ by means of the substitution from 22]. Going further, one might also encode a more clever construction by Buszkowski 8 in order to restrict ourselves further to the product-free onedivision fragment. Finally, one could adopt substitutions from [18] and obtain undecidability for the language with only one variable.

There are also several other linguistically motivated extensions of the Lambek calculus (see, for instance, 30] 28] 32]) and their algorithmic and logical properties should be investigated. 


\section{References}

1. V. M. Abrusci. A comparison between Lambek syntactic calculus and intuitionistic linear propositional logic. Zeitschr. für math. Log. Grundl. Math. (Math. Logic Quart.), 36:11-15, 1990.

2. K. Ajdukiewicz. Die syntaktische Konnexität. Studia Philosophica, Vol. 1, 1-27, 1935.

3. Y. Bar-Hillel. A quasi-arithmetical notation for syntactic description. Language, Vol. 29, 47-58, 1953.

4. G. Barry, M. Hepple, N. Leslie, G. Morrill. Proof figures and structural operators for categorial grammar. Proc. 5th Conference of the European Chapter of ACL, Berlin, 1991.

5. T. Braüner, V. de Paiva. Cut elimination for full intuitionstic linear logic. BRICS Report RS-96-10, April 1996.

6. T. Braüner, V. de Paiva. A formulation of linear logic based on dependency relations. Proc. CSL 1997, LNCS vol. 1414, Springer, 1998, 129-148.

7. T. Braüner. A cut-free Gentzen formulation of modal logic S5. Log. J. IGPL, $8(5): 629-643,2000$.

8. W. Buszkowski. Some decision problems in the theory of syntactic categories. Zeitschr. für math. Logik und Grundl. der Math. (Math. Logic Quart.), Vol. 28, 539-548, 1982.

9. W. Buszkowski. Type logics in grammar. In: Trends in Logic: 50 Years of Studia Logica, Springer, 2003, 337-382.

10. W. Buszkowski. Lambek calculus with nonlogical axioms. Language and Grammar. CSLI Lect. Notes vol. 168, 2005, 77-93.

11. B. Carpenter. Type-logical semantics. MIT Press, 1997.

12. M. Dekhtyar, A. Dikovsky. Generalized categorial dependency grammars. Trakhtenbrot/Festschrift, LNCS vol. 4800, Springer, 2008, 230-255.

13. H. Eades III, V. de Paiva. Multiple conclusion linear logic: cut elimination and more. Proc. LFCS 2016. LNCS vol. 9537, 2015, 90-105.

14. G. Gentzen. Untersuchungen über das logische Schließen I. Mathematische Zeitschrift, Vol. 39, 176-210, 1935.

15. J.-Y. Girard. Linear logic. Theor. Comput. Sci. 50:1-102, 1987.

16. Ph. de Groote. On the expressive power of the Lambek calculus extended with a structural modality. Language and Grammar. CSLI Lect. Notes, vol. 168, 2005, 95-111.

17. M. Kanazawa. Lambek calculus: Recognizing power and complexity. In: J. Gerbrandy et al. (eds.). JFAK. Essays dedicated to Johan van Benthem on the occasion of his 50th birthday. Vossiuspers, Amsterdam Univ. Press, 1999.

18. M. Kanovich. The complexity of neutrals in linear logic. Proc. LICS '95, 1995, 486-495.

19. M. Kanovich, S. Kuznetsov, A. Scedrov. On Lambek's restriction in the presence of exponential modalities. Proc. LFCS '16. LNCS vol. 9537, 2015, 146-158.

20. M. Kanovich, S. Kuznetsov, A. Scedrov. Undecidability of the Lambek calculus with a relevant modality. Proc. FG '15 and FG' 16 . LNCS vol. 9804, 2016, 240-256 (arXiv: 1601.06303).

21. M. Kanovich, S. Kuznetsov, G. Morrill, A. Scedrov. A polynomial time algorithm for the Lambek calculus with brackets of bounded order. arXiv preprint 1705.00694, 2017. Submitted for publication. 
22. S. L. Kuznetsov. On the Lambek calculus with a unit and one division. Moscow Univ. Math. Bull., 66:4 (2011), 173-175.

23. J. Lambek. The mathematics of sentence structure. Amer. Math. Monthly, Vol. 65, No. 3, 154-170, 1958.

24. J. Lambek. Deductive systems and categories II: Standard constructions and closed categories. Category Theory, Homology Theory and their Applications I. Lect. Notes Math. vol. 86, Springer, 1969, 76-122.

25. P. Lincoln, J. Mitchell, A. Scedrov, N. Shankar. Decision problems for propositional linear logic. APAL, 56:239-311, 1992.

26. A. Markov. On the impossibility of certain algorithms in the theory of associative systems. Doklady Acad. Sci. USSR (N. S.), 55 (1947), 583-586.

27. M. Moortgat. Multimodal linguistic inference. J. Log. Lang. Inform., 5(3,4):349385, 1996.

28. R. Moot, C. Retoré. The logic of categorial grammars: a deductive account of natural language syntax and semantics. Springer, 2012.

29. G. Morrill. Categorial formalisation of relativisation: pied piping, islands, and extraction sites. Technical Report LSI-92-23-R, Universitat Politècnica de Catalunya, 1992.

30. G. V. Morrill. Categorial grammar: logical syntax, semantics, and processing. Oxford University Press, 2011.

31. G. Morrill. CatLog: a categorial parser/theorem-prover. System demonstration, LACL 2012, Nantes, 2012.

32. G. Morrill. Grammar logicised: relativisation. Linguistics and Philosophy, 40(2): 119-163, 2017.

33. G. Morrill, O. Valentín. Computational coverage of TLG: Nonlinearity. Proc. NLCS '15 (EPiC Series, vol. 32), 2015. P. 51-63.

34. V. Nigam, D. Miller. Algorithmic specifications in linear logic with subexponentials. Proc. PPDP '09, ACM, 2009. P. 129-140.

35. M. Pentus. Lambek calculus is NP-complete. Theor. Comput. Sci. 357(1):186-201, 2006.

36. M. Pentus. A polynomial time algorithm for Lambek grammars of bounded order. Linguistic Analysis, 36(1-4):441-471, 2010.

37. E. L. Post. Recursive unsolvability of a problem of Thue. J. Symb. Log., 12 (1947), 1-11.

38. J. R. Ross. Constraints on variables in syntax. Ph. D. Thesis, MIT, 1967.

39. M. Steedman. The syntactic process. MIT Press, 2000.

40. A. Thue. Probleme über Veränderungen von Zeichenreihen nach gegebener Regeln. Kra. Vidensk. Selsk. Skrifter., 10, 1914. (In: Selected Math. Papers, Univ. Forlaget, Oslo, 1977, pp. 493-524.) 


\section{Appendix I. Cut Elimination Proof for $!_{b} L^{1}$}

In this section we give a complete proof of Lemma 1, which is the main step of cut elimination in $!_{\mathrm{b}} \mathbf{L}^{\mathbf{1}}$ (Theorem 1).

Proof. Proceed by nested induction on two parameters:

1. The complexity $\kappa$ of the formula $A$ being cut.

2. The total number $\sigma$ of rule applications in $\mathscr{D}_{\text {left }}$ and $\mathscr{D}_{\text {right }}$.

In each case either $\kappa$ gets reduced, or $\kappa$ remains the same and $\sigma$ gets reduced.

Case 1 (axiomatic). One of the premises of (cut) is an axiom of the form $A \rightarrow A$. Then the other premise coincides with the goal, and cut disappears.

Case 2 (left non-principal).

Subcase 2.a. The last rule in $\mathscr{D}_{\text {left }}$ is one of the one-premise rules operating only on the left-hand side of the sequent: $(\cdot \rightarrow),(\mathbf{1} \rightarrow),\left([]^{-1} \rightarrow\right),(\langle\rangle \rightarrow),(! \rightarrow)$, $\left(\right.$ perm $\left._{i}\right),($ contr). Denote this rule by $(R)$. Notice that $(R)$ can be applied in any context, and transform the derivation in the following way:

$$
\frac{\frac{\Pi^{\prime} \rightarrow A}{\Pi \rightarrow A}(R) \Delta(A) \rightarrow C}{\Delta(\Pi) \rightarrow C}(\text { cut }) \leadsto \frac{\Pi^{\prime} \rightarrow A \quad \Delta(A) \rightarrow C}{\frac{\Delta\left(\Pi^{\prime}\right) \rightarrow C}{\Delta(\Pi) \rightarrow C}(R)} \text { (cut) }
$$

The $\sigma$ parameter gets reduced, therefore the new cut is eliminable by induction hypothesis.

Subcase 2.b. The last rule in $\mathscr{D}_{\text {left }}$ is $(\backslash \rightarrow)$ or $(/ \rightarrow)$. Then the derivation fragment

$$
\frac{\frac{\Pi^{\prime} \rightarrow E \quad \Pi(F) \rightarrow A}{\Pi\left(\Pi^{\prime}, E \backslash F\right) \rightarrow A}(\backslash \rightarrow) \quad \Delta(A) \rightarrow C}{\Delta\left(\Pi\left(\Pi^{\prime}, E \backslash F\right)\right) \rightarrow C} \text { (cut) }
$$

is transformed into

$$
\frac{\Pi^{\prime} \rightarrow E}{\Delta\left(\Pi\left(\Pi^{\prime}, E \backslash F\right)\right) \rightarrow C}(\backslash \rightarrow)
$$

Again, $\sigma$ decreases. The $(/ \rightarrow)$ case is handled symmetrically.

Case 3 (deep). The last rule applied on the left is $(\rightarrow !)$. Then the cut rule application has the following form:

$$
\frac{\frac{! \Pi \rightarrow A}{! \Pi \rightarrow ! A}(\rightarrow !) \quad \Delta\langle! A\rangle \rightarrow C}{\Delta\langle! \Pi\rangle \rightarrow C}(\mathrm{cut})
$$

The right premise, $\Delta\langle! A\rangle \rightarrow C$, has a cut-free derivation tree $\mathscr{D}_{\text {right }}$. Let us trace the designated occurrence of $! A$ in $\mathscr{D}_{\text {right }}$. The trace can branch if (contr) 
is applied to this formula. Each branch of the trace ends either with an axiom $(! A \rightarrow ! A)$ leaf or with an application of $(! \rightarrow)$ that introduces $! A$.

The axiom $! A \rightarrow ! A$ can be reduced to $A \rightarrow A$ by consequent application of $(! \rightarrow)$ and $(\rightarrow !)$. Therefore, without loss of generality, we can assume that all branches lead to applications of $(! \rightarrow)$. The whole picture is shown on Figure 1 .

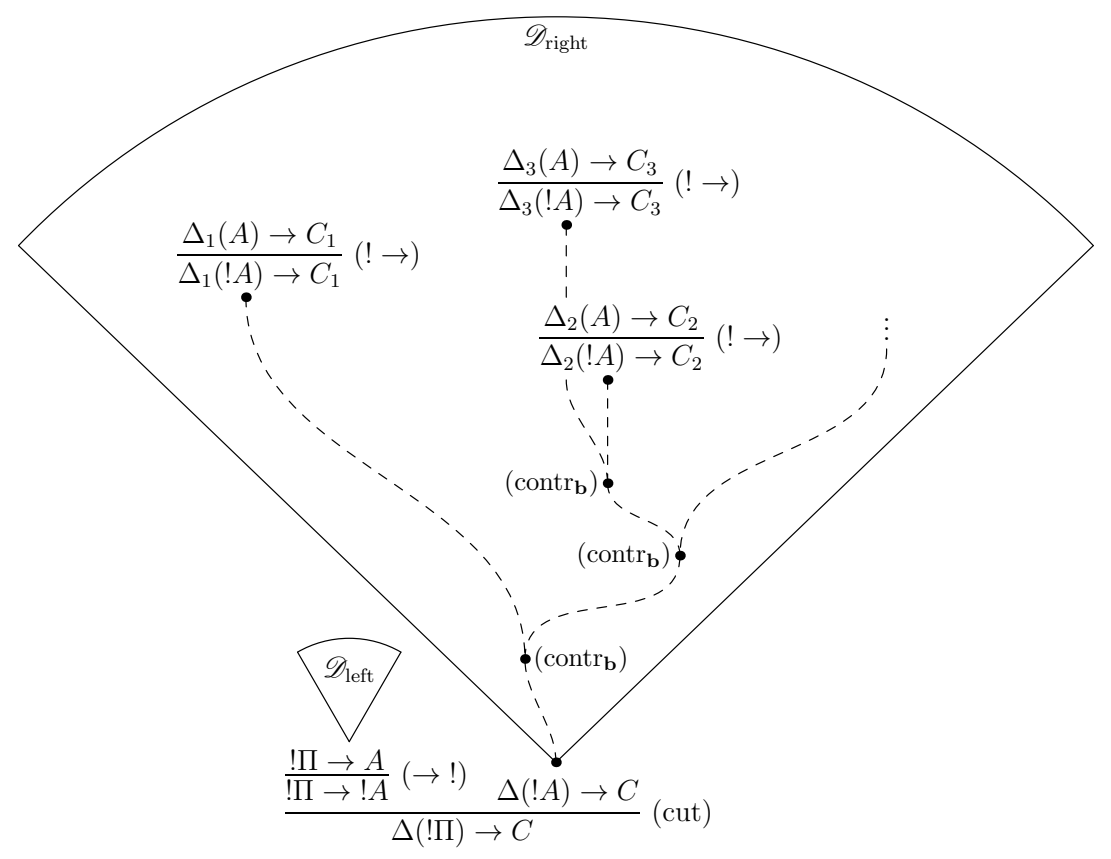

Fig. 1.

In $\mathscr{D}_{\text {right }}$ we replace the designated occurrences of $! A$ with $! \Pi$ along the traces. The applications of (contr) remain valid; if there were permutation rules applied, we replace such a rule with a series of permutations for each formula in ! $\Pi$. Other rules do not operate ! $A$ and therefore remain intact. After this replacement applications of $(! \rightarrow)$ tranform into applications of (cut) with $\Pi \rightarrow A$ as the left premise (Figure 2). One case could go through several instances of $(! \rightarrow$ ) with the active ! $A$, like $\Delta_{2}$ and $\Delta_{3}$ in the example; in this case we go from top to bottom.

The new cuts have lower $\kappa$ (the cut formula is $A$ instead of $! A$ ), and therefore they are eliminable by induction hypothesis.

Case 4 (principal). In the so-called principal case, the last rules both in $\mathscr{D}_{\text {left }}$ and in $\mathscr{D}_{\text {right }}$ introduce the main connective of the formula $A$ being cut. Note that $A$ here is not of the form $! A^{\prime}$ (this is the previous case). In the principal case, the $\kappa$ parameter gets reduced, and therefore the induction hypothesis can be applied to eliminate the new cut(s) that arise after the transformation. 


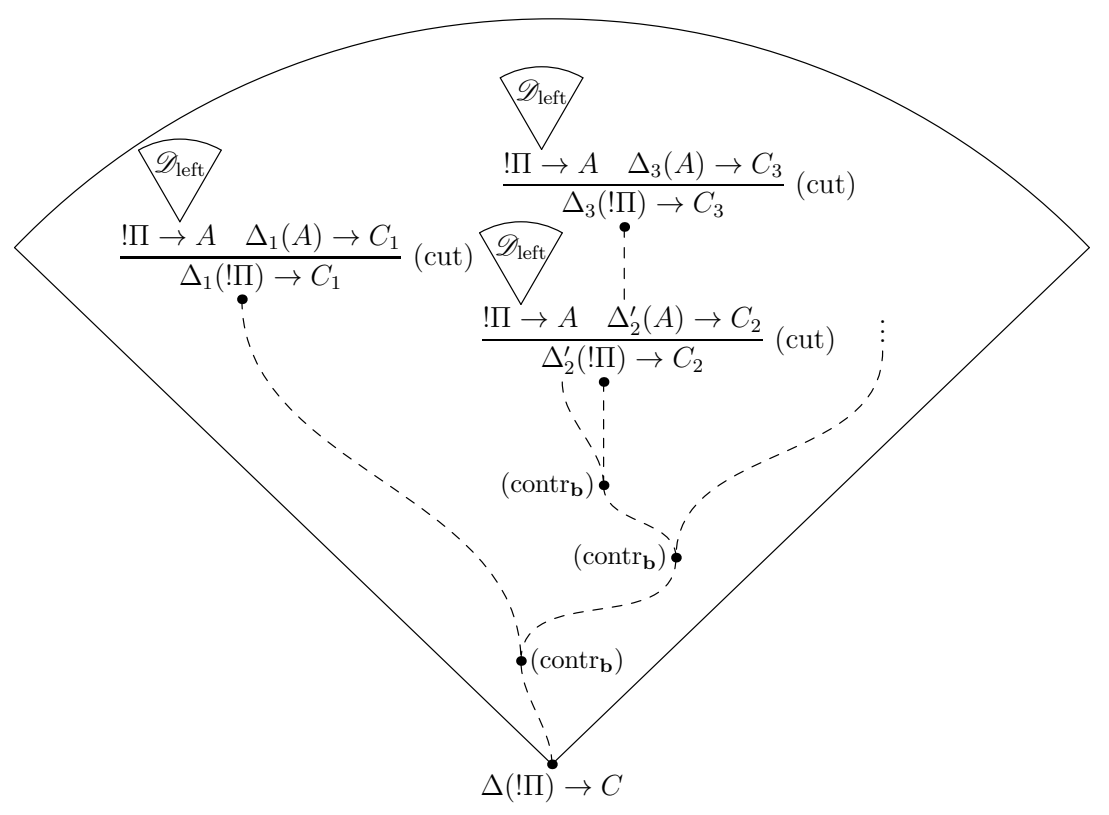

Fig. 2.

Subcase 4.a: $(\rightarrow \backslash)$ vs. $(\backslash \rightarrow)$ or $(\rightarrow /)$ vs. $(/ \rightarrow)$. In this case $A=A_{1} \backslash A_{2}$ (the / case is handled symmetrically), and the derivation fragment

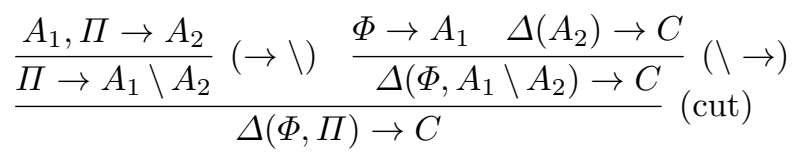

transforms into

$$
\frac{\Phi \rightarrow A_{1} \frac{A_{1}, \Pi \rightarrow A_{2} \quad \Delta\left(A_{2}\right) \rightarrow C}{\Delta\left(A_{1}, \Pi\right) \rightarrow C} \text { (cut) }}{\Delta(\Phi, \Pi) \rightarrow C} \text { (cut) }
$$

Subcase 4.b. $(\rightarrow \cdot)$ vs. $(\cdot \rightarrow)$. In this case $A=A_{1} \cdot A_{2}$, and the derivation fragment

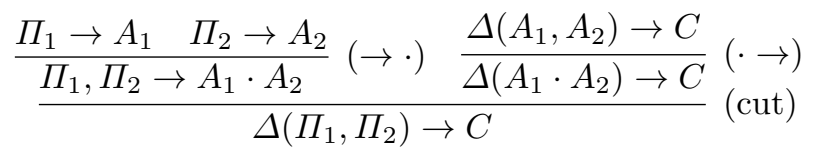

transforms into

$$
\frac{\Pi_{2} \rightarrow A_{2} \frac{\Pi_{1} \rightarrow A_{1} \quad \Delta\left(A_{1}, A_{2}\right) \rightarrow C}{\Delta\left(\Pi_{1}, A_{2}\right) \rightarrow C} \text { (cut) }}{\Delta\left(\Pi_{1}, \Pi_{2}\right) \rightarrow C} \text { (cut) }
$$


Subcase 4.c. $(\rightarrow \mathbf{1})$ vs. $(\mathbf{1} \rightarrow)$. In this case $A=\mathbf{1}$ :

$$
\frac{\overline{\Lambda \rightarrow \mathbf{1}}(\rightarrow \mathbf{1}) \frac{\Delta(\Lambda) \rightarrow C}{\Delta(\mathbf{1}) \rightarrow C}(\mathbf{1} \rightarrow)}{\Delta(\Lambda) \rightarrow C}(\text { cut })
$$

The cut disappears, since its goal coincides with the premise of $(\mathbf{1} \rightarrow)$.

Subcase 4.d. $\left(\rightarrow[]^{-1}\right)$ vs. $\left([]^{-1} \rightarrow\right)$. In this case $A=[]^{-1} A^{\prime}$, and the derivation fragment

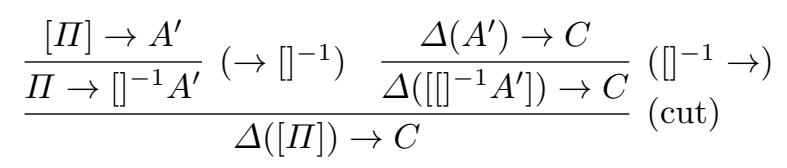

transforms into

$$
\frac{[\Pi] \rightarrow A^{\prime} \quad \Delta\left(A^{\prime}\right) \rightarrow C}{\Delta([\Pi]) \rightarrow C}(\mathrm{cut})
$$

Subcase 4.e. $(\rightarrow\langle\rangle)$ vs. $(\langle\rangle \rightarrow)$. In this case $A=\langle\rangle A^{\prime}$, and the derivation fragment

$$
\frac{\frac{\Pi \Pi \rightarrow A^{\prime}}{[\Pi] \rightarrow\langle\rangle A^{\prime}}(\rightarrow\langle\rangle) \frac{\Delta\left(\left[A^{\prime}\right]\right) \rightarrow C}{\Delta\left(\langle\rangle A^{\prime} \rightarrow C\right.}(\langle\rangle \rightarrow)}{\Delta([\Pi]) \rightarrow C}(\text { cut })
$$

transforms into

$$
\frac{\Pi \rightarrow A^{\prime} \quad \Delta\left(\left[A^{\prime}\right]\right) \rightarrow C}{\Delta([\Pi]) \rightarrow C} \text { (cut) }
$$

Case 5 (right non-principal). In the remaining cases, $A$ is not of the form $! A^{\prime}$ (therefore the last rule of $\mathscr{D}_{\text {right }}$ is not $(\rightarrow !)$; it is also not $(\rightarrow \mathbf{1})$, since there is nothing to cut in an empty antecedent) and the last rule of $\mathscr{D}_{\text {right }}$ does not operate on $A$. In this case, the cut gets propagated upwards to $\mathscr{D}_{\text {right }}$, decreasing $\sigma$ with the same $\kappa$.

\section{Appendix II. Axioms and Rules of ! $^{1}$}

$$
\begin{aligned}
& \overline{A \rightarrow A} \quad \overline{\Lambda \rightarrow 1} \\
& \frac{\Gamma \rightarrow B \quad \Delta_{1}, C, \Delta_{2} \rightarrow D}{\Delta_{1}, C / B, \Gamma, \Delta_{2} \rightarrow D}(/ \rightarrow) \frac{\Gamma, B \rightarrow C}{\Gamma \rightarrow C / B}(\rightarrow /) \frac{\Delta_{1}, A, B, \Delta_{2} \rightarrow D}{\Delta_{1}, A \cdot B, \Delta_{2} \rightarrow D}(\cdot \rightarrow) \\
& \frac{\Gamma \rightarrow A \quad \Delta_{1}, C, \Delta_{2} \rightarrow D}{\Delta_{1}, \Gamma, A \backslash C, \Delta_{2} \rightarrow D}(\backslash \rightarrow) \frac{A, \Gamma \rightarrow C}{\Gamma \rightarrow A \backslash C}(\rightarrow \backslash) \frac{\Gamma_{1} \rightarrow A \quad \Gamma_{2} \rightarrow B}{\Gamma_{1}, \Gamma_{2} \rightarrow A \cdot B}(\rightarrow \cdot) \\
& \frac{\Gamma_{1}, A, \Gamma_{2} \rightarrow B}{\Gamma_{1}, ! A, \Gamma_{2} \rightarrow B}(! \rightarrow) \quad \frac{\Delta_{1}, ! A, \Gamma, \Delta_{2} \rightarrow B}{\Delta_{1}, \Gamma, ! A, \Delta_{2} \rightarrow B}\left(\operatorname{perm}_{1}\right) \quad \frac{\Delta_{1}, \Gamma, ! A, \Delta_{2} \rightarrow B}{\Delta_{1}, ! A, \Gamma, \Delta_{2} \rightarrow B}\left(\operatorname{perm}_{2}\right) \\
& \frac{\Delta_{1}, \Delta_{2} \rightarrow A}{\Delta_{1}, \mathbf{1}, \Delta_{2} \rightarrow A}(\mathbf{1} \rightarrow) \frac{! A_{1}, \ldots, ! A_{n} \rightarrow A}{! A_{1}, \ldots, ! A_{n} \rightarrow ! A}(\rightarrow !) \frac{\Delta_{1}, ! A, ! A, \Delta_{2} \rightarrow B}{\Delta_{1}, ! A, \Delta_{2} \rightarrow B} \text { (contr) } \\
& \frac{\Pi \rightarrow A \quad \Delta_{1}, A, \Delta_{2} \rightarrow C}{\Delta_{1}, \Pi, \Delta_{2} \rightarrow C} \text { (cut) }
\end{aligned}
$$

